\title{
Cloning and characterization of the $U B C$ gene from lotus (Nelumbo nucifera)
}

\author{
Y. Diao ${ }^{1 *}$, G.L. Li ${ }^{2 *}$, A.Q. Yu ${ }^{2}$, X.W. Zheng ${ }^{3}$, K.Q. Xie ${ }^{3}$, Y.W. Wang ${ }^{4}$, \\ M.Q. Zhou ${ }^{5}$, J. Ming ${ }^{6}$ and Z.L. Hu ${ }^{2}$ \\ ${ }^{1}$ College of Forestry and Life Sciences, \\ Chongqing University of Arts and Sciences, Chongqing, China \\ ${ }^{2}$ State Key Laboratory of Hybrid Rice, College of Life Sciences, \\ Wuhan University, Wuhan, Hubei Province, China \\ ${ }^{3}$ White Lotus Research Institute of Guangchang, Guangchang, \\ Jiangxi Province, China \\ ${ }^{4}$ School of Pharmaceutical Sciences, Wuhan University, \\ Wuhan, Hubei Province, China \\ ${ }^{5}$ Lotus Engineering Research Center of Hubei Province, \\ Wuhan University, Wuhan, Hubei Province, China \\ ${ }^{6}$ Institute of Vegetables and Flowers, \\ Chinese Academy of Agricultural Sciences, Beijing, China \\ *These authors contributed equally to this study. \\ Corresponding authors: J. Ming / Z.L. Hu \\ E-mail: mingjun@caas.cn / huzhongli@whu.edu.cn
}

Genet. Mol. Res. 15 (3): gmr.15038341

Received August 8, 2015

Accepted November 26, 2015

Published July 29, 2016

DOI http://dx.doi.org/10.4238/gmr.15038341

Copyright $(C 2016$ The Authors. This is an open-access article distributed under the terms of the Creative Commons Attribution ShareAlike (CC BY-SA) 4.0 License.

ABSTRACT. Protein ubiquitination is extensively involved in the
regulation of a considerable number of physiological processes in plant
cells. E2 (ubiquitin-conjugating enzyme, UBC), one of the essential
enzymes of eukaryotic ubiquitination, catalyzes protein ubiquitination
together with E1 and E3. In this study, we cloned four full-length cDNA

Genetics and Molecular Research 15 (3): gmr.15038341 
NnUBCs of Nelumbo nucifera. With the same coding sequence length of $459 \mathrm{bp}$ and coding 153 amino acids, these four genes are highly homologous with the AtUBC1 and AtUBC2 of Arabidopsis thaliana. Quantitative fluorescence polymerase chain reaction showed that these four genes exhibited different expression patterns in different tissues of $N$. nucifera. Overall, the expression of $N n U B C 3$ was the highest in all plant tissues. Tests of different stress treatments showed that NnUBC3 plays an important role in response to heat, salt, and drought stresses in N. nucifera. Moreover, transgenic Arabidopsis plants (Atubc1-1Atubc2-1 mutant) expressing NnUBC3 presented a wild-type phenotype, indicating that $\mathrm{NnUBC} 3$ performs the same function as AtUBC1 and AtUBC2.

Key words: Nelumbo nucifera; $U B C$ gene; cDNA; Expression; Transformation experiment

\section{INTRODUCTION}

Protein ubiquitination is extensively involved in the regulation of a considerable number of physiological processes in plant cells. These include hormonal signal transduction, flower development, photoperiodism, photomorphogenesis, aging, immunity, and plant self-incompatibility (Smalle and Vierstra, 2004; Liu et al., 2007; Bartel and Citovsky, 2012; Cheng and Li, 2012; Maier et al., 2013). These processes are mainly catalyzed by a cascade of enzymes, including E1 (ubiquitin-activating enzyme, UBA), E2 (ubiquitin-conjugating enzyme, UBC), and E3 (ubiquitin ligase, UBL) (Scheffner et al., 1995). UBC has the same active site as UBA and UBL; thus, UBC plays the role of a bridge between UBA and UBL in the protein ubiquitination reaction (Pickart, 2001). UBC is a large family of proteins with several members. UBC carries ubiquitin, and identifies the target protein in the ubiquitin chain. Different UBCs mediate different ubiquitination roles. Current studies on UBC have mainly focused on model organisms. For example, $11 U B C$ genes have been found in the yeast genome (Bachmair et al., 2001); at least 37 UBC genes have been reported in Arabidopsis thaliana that are divided into 12 subgroups (Kraft et al., 2005). In addition, there are 48 putative UBCs in rice and the mode of ubiquitination of SPL11 UBL is critically influenced by individual UBCs (Bae and Kim, 2014).

Lotus (Nelumbo nucifera L.) belongs to Nelumbonaceae, Nymphaeales, and is a perennial aquatic plant. It is one of the oldest dicotyledonous plants and has a number of monocotyledon characteristics (Esau and Kosakai, 1975). Lotus is native to tropical and temperate regions of Asia (Borsch and Barthlott, 1994). According to historical records, the lotus has been cultivated as an ornamental plant and vegetable since the Chow Dynasty in China (Sridhar and Bhat, 2007). Moreover, nearly each part of lotus has been used in Chinese traditional medicine, including the seeds, roots, leaves, flowers, and embryos. $N$. nucifera propagates by seed or rhizome. Notably, its seeds can survive for thousands of years, making it the oldest viable seed for which an age has been directly determined (Priestley and Posthumus, 1982). To date, the entire genomic sequence of $N$. nucifera has been published (Ming et al., 2013); however, the UBC gene has not yet been reported. Therefore, in this study, the UBC gene was cloned and characterized in $N$. nucifera. We used over-expression of the lotus UBC gene in A. thaliana to understand its biological functions in N. nucifera.

Genetics and Molecular Research 15 (3): gmr.15038341 


\section{MATERIAL AND METHODS}

\section{Plant materials and stress treatments}

The experimental material was $N$. nucifera $\mathrm{cv}$. Honghua Jianlian, which was planted in a greenhouse at Wuhan University. Seedlings were allowed to grow in the greenhouse for 10 days at $24^{\circ} \mathrm{C}$ and were then treated with cold stress $\left(0,1.5,3.0\right.$, and $6.0 \mathrm{~h}$ at $\left.4^{\circ} \mathrm{C}\right)$, heat stress $\left(0,1.5,3.0\right.$, and $6.0 \mathrm{~h}$ at $\left.42^{\circ} \mathrm{C}\right)$, mechanical damage (leaves were scratched with blades and samples of the damaged leaves were taken $0,1.5,3.0$, and $6 \mathrm{~h}$ later), and three chemical stress treatments ( $6.0 \mathrm{~h}$ in $200 \mathrm{mM} \mathrm{NaCl}, 20 \% \mathrm{PEG}$, or $50 \mu \mathrm{M} \mathrm{ABA})$. Fresh leaves were collected for further studies. When flower buds had appeared, after 2 weeks, the leaves, rhizomes, terminal buds, and infant flowers were collected for further analysis.

Seeds of $A$. thaliana were kindly provided by Prof. Shen Wenhui (the National Center for Scientific Research of France), including the wild type (Columbia Col-0) and the double mutant Atubc1-1Atubc2-1. The defining phenotype is a dramatically reduced number of rosette leaves and an early-flowering phenotype (Xu et al., 2009). All A. thaliana materials used in this study were grown under long-day conditions (16-h light, 8-h dark) in a greenhouse at $24^{\circ} \mathrm{C}$.

\section{Extraction of RNA and synthesis of cDNA}

Total RNA was isolated from fresh materials using the RNAprep Plant Kit (Tiangen Biotech Co., Ltd., Beijing, China). For synthesis of first-strand cDNA, the RNA was first incubated for $5 \mathrm{~min}$ at $70^{\circ} \mathrm{C}$ with oligo(dT)-m13 (Table 1), and then reversed to cDNA using M-MLV Reverse Transcriptase (Promega Corporation, Madison, WI, USA). The cDNA was diluted and stored at $-20^{\circ} \mathrm{C}$ until further use.

\begin{tabular}{|c|c|}
\hline Primer & Sequence \\
\hline oligo(dT)-m13 & 5'-GTTTTCCCAGTCACGACTTTTTTTTTTTTTTTTTTTTVN-3' \\
\hline F1 & 5'-ATGTCRACBCCNGCWMGG-3' \\
\hline $\mathrm{R} 1$ & 5'-CTAGTCNGCHGTCCARCT-3' \\
\hline AAP & 5'-GGCCACGCGTCGACTAGTACGGGIIGGGIIGGGIIG-3' \\
\hline GSP1 & 5'-GTTCAACAATTTCACG-3' \\
\hline AUAP & 5'-GGCCACGCGTCGACTAGTAC-3' \\
\hline M13 & 5'-GTTTTCCCAGTCACGAC-3' \\
\hline GPS2 & 5'-ATGTTTAGTGAGAACAA-3' \\
\hline NnUBC1 F & 5'-ACTGATGACAATTAACGC-3' \\
\hline NnUBC1 R & 5'-CTCAAGGATAAGAAACGTG-3' \\
\hline NnUBC2 F & 5'-CATCAATTCATCATCTTGATG-3' \\
\hline NnUBC2 R & 5'-CATAAAAGATGCCAACATAC-3' \\
\hline NnUBC3 F & 5'-GCTATGTAGTATGTACATG-3' \\
\hline NnUBC3 R & 5'-GAAGTTAAAGGGAGTTAGC-3' \\
\hline NnUBC4 F & 5'-TTGAGAATGCCCGAGTAGTGTT-3' \\
\hline NnUBC4 R & 5'-AAGCATCCAAAACACCCTACC-3' \\
\hline NnActin F & 5'-TGCAGGAATCCATGAGACTACC-3' \\
\hline NnActin R & 5'-GGTCAGCAATACCAGGGAACAT-3' \\
\hline AtActin F & 5'-GGAAGGATCTGTACGGTAAC-3' \\
\hline AtActin R & 5'-TGTGAACGATTCCTGGACCT-3' \\
\hline
\end{tabular}

Full-length cDNA isolation by real-time polymerase chain reaction (RT-PCR) and RACE

The full-length cDNA was divided into three amplifiable fragments. Based on the

Genetics and Molecular Research 15 (3): gmr.15038341 
conservative region of the plant $U B C$ genes, a degenerate primer pair F1 and R1 (Table 1) was designed to amplify partial cDNA of the lotus $U B C$ gene $(N n U B C)$, using the first-strand cDNA as template. SMART-RACE technology was used for amplification of 3'- and 5'-RACE. The 5'-RACE was generated by nested PCR using the primary primer set of AAP and GSP1, and the second set of the 5'-nested primer AUAP and GSP1 (Table 1). Similarly, the 3'-RACE was generated by the 3 '-primer set M13 and GPS2 (Table 1). The PCR product was ligated into pGEM-T vector (Promega Corporation), transformed into Escherichia coli DH5a, and then sequenced.

\section{Analysis of gene expression}

Total RNA was extracted from different tissues (leaf, rhizome, terminal bud, and flower) and reversed to cDNA. Specific primer pairs of each NnUBC gene was designed based on their 3'-UTR sequences, which were NnUBC1 F and NnUBC1 R for NnUBC1; NnUBC2 F and NnUBC2 R for NnUBC2; NnUBC3 F and NnUBC3 R for NnUBC3; and NnUBC4 F and NnUBC4 R for NnUBC4 (Table 1). The $\beta$-actin of $N$. nucifera (EU131153) was used as a reference gene, in which the designed primers were NnActin F and NnActin R (Table 1). RT-PCR was carried out in a fluorimetric thermal cycler (Rotor Gene 2000; Corbett Research, Sydney, Australia) using the DNA-binding dye SYBR Green I (Toyobo Co., Ltd., Osaka, Japan) for detection of PCR products. The reaction was conducted with SYBR Green real-time PCR Master Mix according to the manufacturer instructions. The PCR procedure was as follows: initial denaturation at $94^{\circ} \mathrm{C}$ for $3 \mathrm{~min}$; followed by 50 cycles at $94^{\circ} \mathrm{C}$ for $10 \mathrm{~s}$ and $60^{\circ} \mathrm{C}$ for $20 \mathrm{~s}$. Each reaction included three replicates, and the trials were repeated three times. Semi-quantitative data analysis was carried out according to the $2^{-\Delta \Delta \mathrm{Ct}}$ method published by Livak and Schmittgen (2001).

\section{Agrobacterium-mediated transformation to $A$. thaliana}

The Agrobacterium GV3101 containing the expression vector was used for the transformation experiments. The expression vector was transferred to A. thaliana (Atubcl1Atubc2-1 mutant) using the floral dip method (Clough and Bent, 1998). The transformed A. thaliana plants and wild types were continuously cultured in the greenhouse at $22^{\circ} \mathrm{C}$ under long-day conditions (16-h light: 8-h dark). The concentration of the selected antibiotic, hygromycin, was $50 \mathrm{mg} / \mathrm{L}$. Individuals from the T3-generation were used in further tests. RNA of 2-week-old seedlings was extracted from the mutant and T3 plant. The expression level of NnUBC3 was detected by qRT-PCR, with Actin as reference gene (Table 1).

\section{RESULTS}

\section{Characterization of UBC cDNA in $N$. nucifera}

cDNA of four $U B C$ genes in $N$. nucifera were obtained with full-length coding sequence (CDS) and 3'-UTR sequence, using RACE technology. The CDS regions of the four cDNAs had the same length of $459 \mathrm{bp}$, coding for 153 amino acids. The four cDNAs had the conserved catalytic site of UBC activity C88. Moreover, the similarity of these four genes was $97-99 \%$. However, the 3'-UTR sequences were significantly different among the four

Genetics and Molecular Research 15 (3): gmr.15038341 
cDNAs, which indicated that they originated from four different genes. These four genes were defined as NnUBC1-4, respectively (accession Nos. JF970922.1, JF970923.1, JF970924.1, and JF970925.1).

The evolutionary relationship between $N n U B C$ and the 37 genes of $A$. thaliana UBC family was assessed by a phylogenetic amino acid-based sequence analysis using MEGA (Figure 1A). The four $N n U B C$ s clustered together, as expected, and they clustered with $A t U B C 1$ and $A t U B C 2$. The amino acid sequence of lotus $U B C$ s had a significant homology with $A t U B C 1$ and $A t U B C 2$ (Figure 1B). The similarity between lotus $U B C$ s and $A t U B C 1$ was 94-97\%, while the similarity between lotus $U B C$ s and $A t U B C 2$ was $95-98 \%$. These results indicate that the four $N n U B C$ s probably have a function similar to $A t U B C 1$ and $A t U B C 2$.
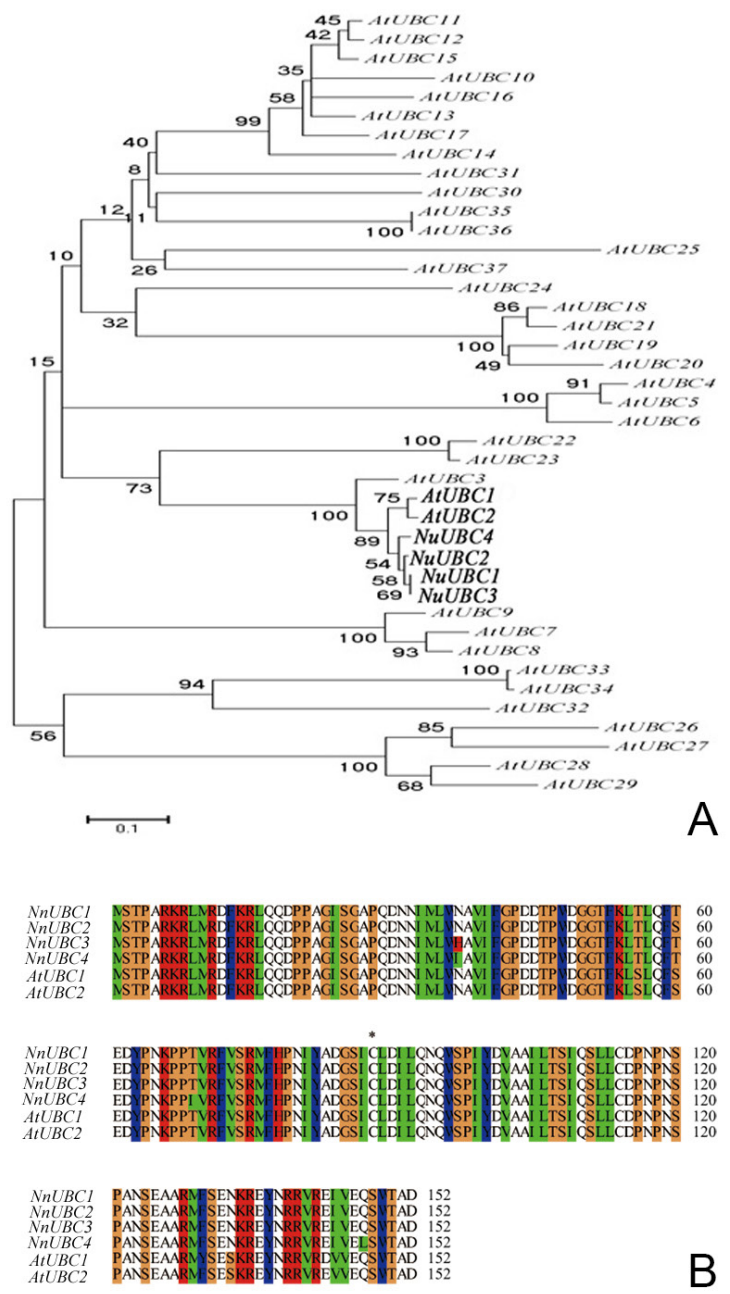

Figure 1. Phylogenetic analysis of NnUBCs with Arabidopsis UBCs. A. Phylogenetic tree. Numbers on branches indicate the percentage of 1000 bootstrap replicates, which support the adjacent node. The unit branch length is 0.1 substitutions per site, as indicated by the scale bar. B. Amino acid sequence comparison of lotus $U B C$ s with $A t U B C 1$ and $A t U B C 2$.

Genetics and Molecular Research 15 (3): gmr.15038341 


\section{Expression analysis of UBC genes in lotus}

The expression levels of the NnUBC genes were detected in four organs; the leaves, stems, apical buds, and flowers (Figure 2). Although, these four genes were expressed in all four organs, their expression levels in leaves and flowers appeared significantly higher than those in stems and terminal buds, except in the case of $N n U B C 2$. Moreover, the expression of each UBC gene varied in different tissues. The expression of $\mathrm{NnUBC1}$ in flowers was higher than in all other tissues. The expression of $N n U B C 2$ in leaves was higher than its expressions in other tissues. Both $N n U B C 3$ and $N n U B C 4$ showed the highest expression levels in leaves and flowers. In general, $N n U B C 3$ showed higher expression in all tissues compared to the other homologs.

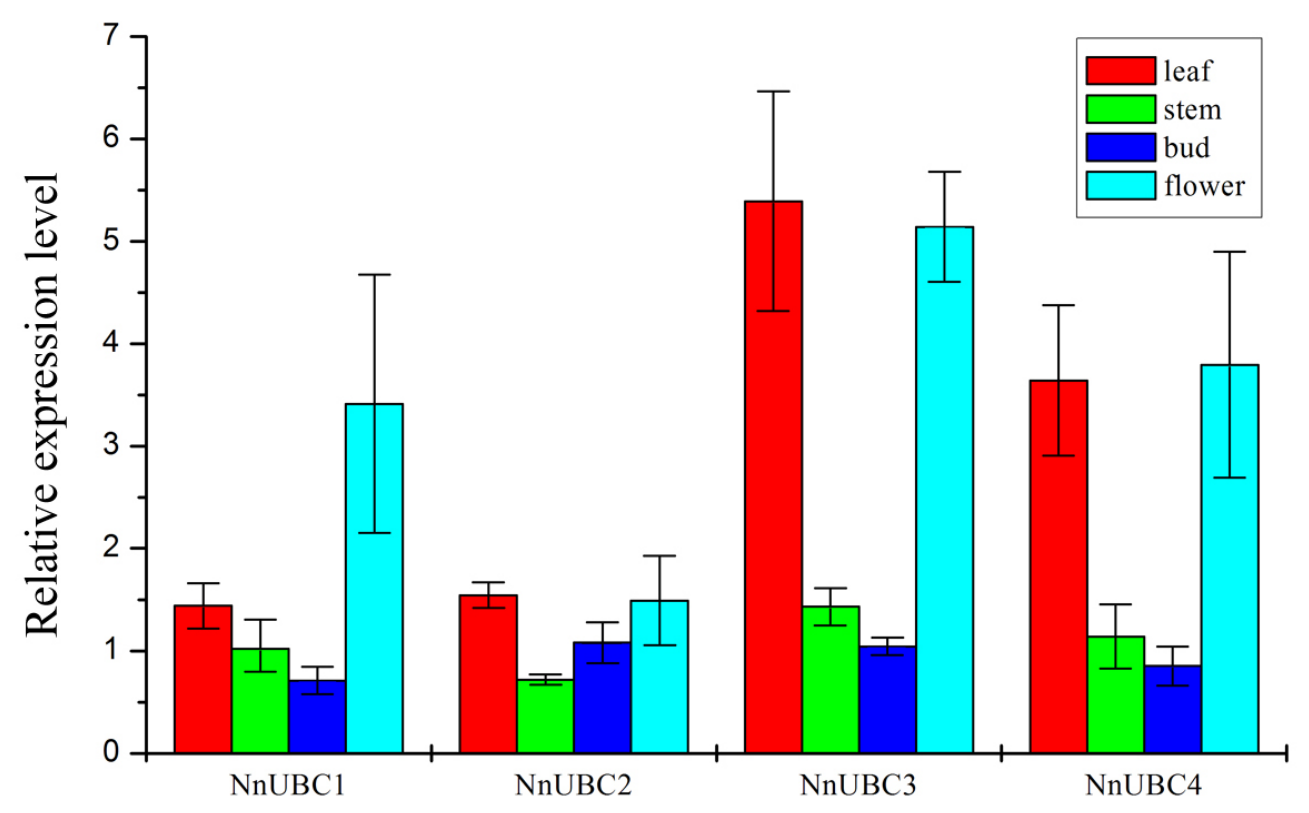

Figure 2. Expression level analysis of NnUBCs in different tissues. Error bars represent the standard deviation of the mean of three replicates.

\section{Structure of the NnUBC3 gene}

Because of the high expression level of $N n U B C 3$ in various tissues, the gene organization of $N n U B C 3$ was further studied. Our results showed that this gene contained four introns and five exons (Figure 3). The gene structure of $N n U B C 3$ was similar to both $A t U B C 1$ and $A t U B C 2$; it had the same number of introns and exons, as well as the insert sites of introns. However, the length of each intron differed between $N n U B C 3$ and $A t U B C 1,2$. For example, the second and third introns in NnUBC 3 were more than 600 bp long, whereas those in $A t U B C 1,2$ were around $200 \mathrm{bp}$.

Genetics and Molecular Research 15 (3): gmr.15038341 
5'UTR

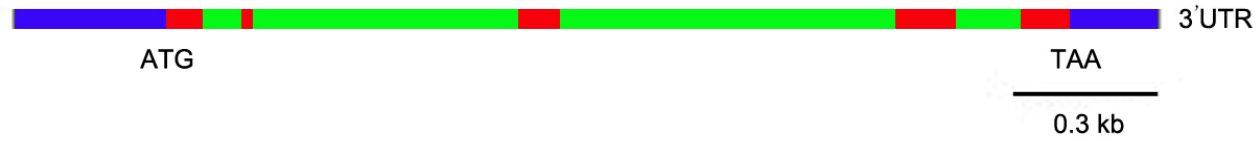

Figure 3. Structure of the Nelumbo nucifera $U B C 3$ gene. Red boxes represented the coding sequences (exons); green boxes represented the noncoding sequences (introns), and blue boxes indicate the 5'- and 3'-untranslated regions. The start (ATG) and the stop (TAA) codons are also indicated.

\section{Expression analysis of $\mathrm{NnUBC} 3$ under different stress conditions}

The expression level of $N n U B C 3$ was the highest among the four identified UBC genes. Therefore, we further analyzed the expression model of $N n U B C 3$ under different stress conditions. After treatment at $4^{\circ} \mathrm{C}$ for $1.5 \mathrm{~h}$, the expression level of NnUBC3 was downregulated approximately $10 \mathrm{X}$ with respect to the negative control, similar to the observed level after treatment for $6.0 \mathrm{~h}$ (Figure $4 \mathrm{~A}$ ). After treatment at $42^{\circ} \mathrm{C}$ for $1.5,3.0$, and $6.0 \mathrm{~h}$, the expression level was down-regulated $10 \mathrm{X}$, down-regulated $5 \mathrm{X}$, and up-regulated $2 \mathrm{X}$, respectively (Figure 4B). The expression of NnUBC3 maintained a low level within $3 \mathrm{~h}$ and recovered after $6 \mathrm{~h}$ in the mechanical damage stress test (Figure 4C). After the treatment of $N$. nucifera with $200 \mathrm{mM} \mathrm{NaCl}, 20 \% \mathrm{PEG}$, or $50 \mu \mathrm{M} \mathrm{ABA}$, the expression levels of NnUBC3 were significantly up-regulated by 120,20 , and $8 X$, respectively (Figure 4D).
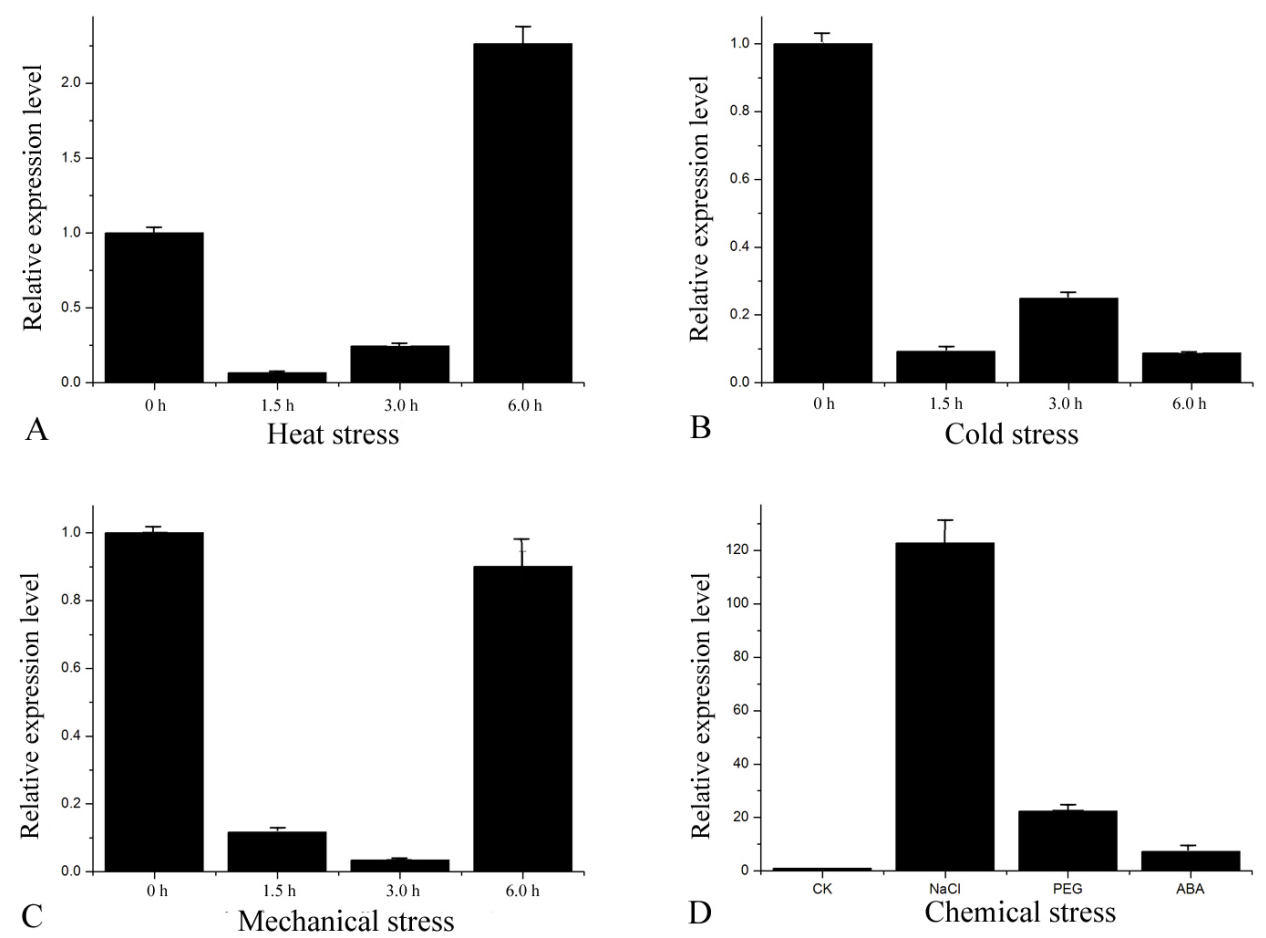

Figure 4. Expression patterns of NnUBC3 under different stress conditions: A. Heat stress. B. Cold stress. C. Mechanical stress. D. Chemical stress. 


\section{Expression of NnUBC3 in transgenic Arabidopsis}

To determine whether the exogenous $N n U B C 3$ gene was integrated into the mutant genome, T3 seedlings of transgenic A. thaliana were analyzed by RT-PCR. As expected, the transgenic plant had an NnUBC3 PCR product, indicating that the $N n U B C 3$ gene had been transferred to the Atubc1-1Atubc2-1 mutant and was successfully expressed (Figure 5A). Under long day conditions, the flowering times of the transgenic, wild-type, and mutant plants were approximately 28,29 , and 20 days, respectively. As expected, after transferring the $N n U B C 3$, the mutant returned to normal flowering time, approaching that of the wild type (Figures 5B and $\mathrm{C}$ ). The number of leaves at flowering of the wild-type, mutant, and transgenic plants was approximately 15,8 , and 15 , respectively (Figure $5 \mathrm{D}$ and $\mathrm{E}$ ).

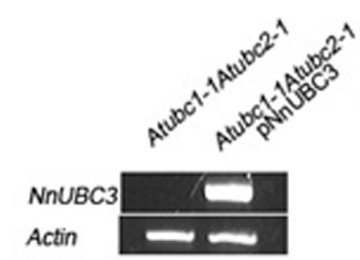

A

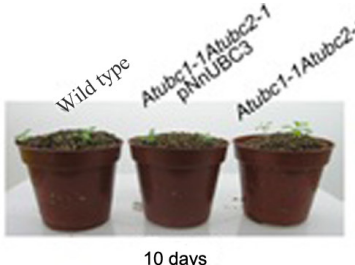

B

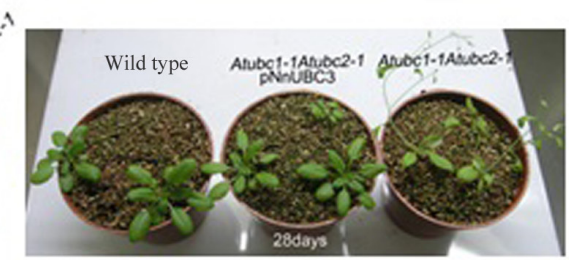

$\mathrm{C}$
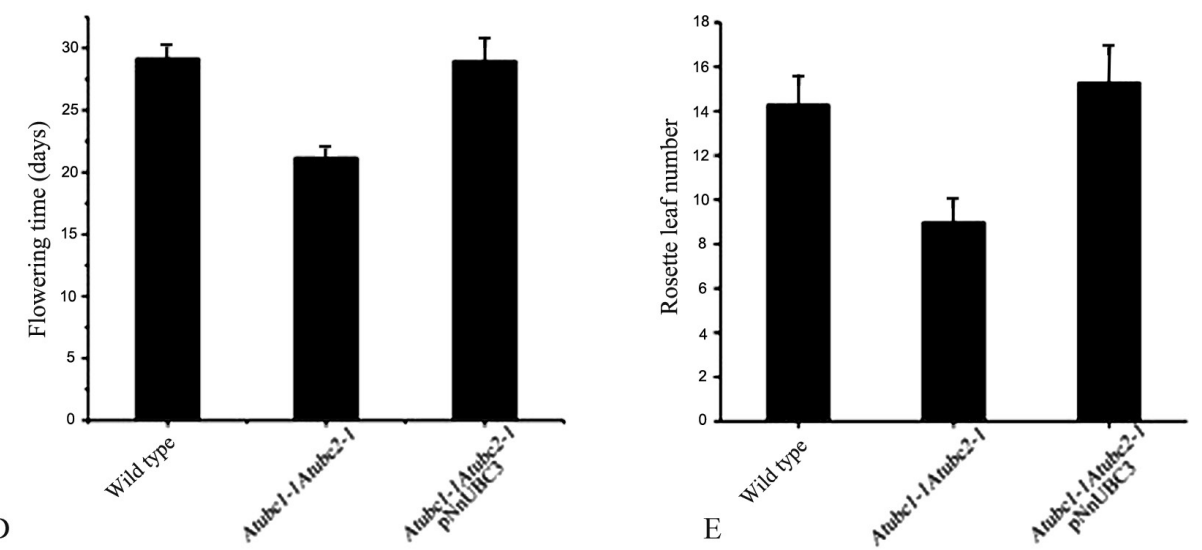

Figure 5. Comparison of wild-type, Atubc1-1Atubc2-1 mutant and transgenic NnUBC3 Arabidopsis plants. A. Identification of mutant and transgenic plants by PCR amplification. B. Wild-type, mutant, and transgenic plants after 10 days growth. C. Wild-type, mutant, and transgenic plants after 28 days growth. D. Flowering time (mean $\pm \mathrm{SE}, \mathrm{N}=3$ ) of wild-type, mutant, and transgenic plants. E. Rosette leaf number (means $\pm \mathrm{SE}, \mathrm{N}$ $=3$ ) of wild-type, mutant, and transgenic plants at flowering.

\section{DISCUSSION}

The UBC proteins play an important role in cell activities, and constitute a protein family with conservative structure, including one UBC domain and one highly conservative catalytic site of cysteine (Dikic et al., 2009; van Wijk and Timmers, 2010). In Arabidopsis, $A t U B C 1$ and $A t U B C 2$ are two highly homologous genes. Previous studies have verified that E2s (AtUBC1 and AtUBC2) and E3s (AtHUB1 and AtHUB2), used for catalyzing H2B 
mono-ubiquitination, have important roles in controlling the flowering time of $A$. thaliana (Cao et al., 2008; Gu et al., 2009; Xu et al., 2009). Detection of H2B ubiquitination of single mutants Atubc1-1, Atubc2-1, and Atubc1-1Atubc2-1 double mutant, revealed that AtUBC1 and $A t U B C 2$ are two functionally redundant genes. The four identified UBC genes in $N$. nucifera are homologous with the $A t U B C 1$ and $A t U B C 2$ genes of $A$. thaliana. However, expression analysis revealed that the four $N$. nucifera genes were expressed in all tissues, indicating that ubiquitination is a basic biological event. However, the expression levels of the genes differed and were significantly tissue-specific, suggesting that the functions of these four UBC genes in $N$. nucifera may not be redundant as the AtUBC1 and $A t U B C 2$ genes are in A. thaliana.

The Atubc1-1Atubc2-1 mutant showed significant defects during both vegetative and reproductive growth. The transgenic line carrying $N n U B C 3$ exhibited the restoration of the Atubc1-1Atubc2-1 phenotype. This indicates that NnUBC3 may execute the UBC function of Atubc1 and Atubc2, mediating H2B ubiquitination to activate floral repressor genes and other processes, as indicated by the pleiotropic phenotypes of the mutants (Xu et al., 2009).

Proteins are damaged when plants are under stress conditions. These damaged proteins pose a significant threat to the plant if not degraded by protein-degrading enzymes in time. Ubiquitin degrades abnormal proteins through the ubiquitin pathway, in response to high temperature, drought, cold damage, radiation, and other forms of stress (Ingvardsen and Veierskov, 2001; Moon et al., 2004; Dreher and Callis, 2007; Shi et al., 2010). Abiotic low-temperature stress on $N$. nucifera resulted in down-regulated $\mathrm{NnUBC3}$ expression. In contrast, the expression level of $N n U B C 3$ was significantly up-regulated when plants were exposed to high temperature, high salt, and drought conditions. This phenomenon indicates that NnUBC3 may be involved in the response of $N$. nucifera to high temperature, high salt, and drought conditions. The expression of this gene was inhibited under low-temperature stress. There may be other ubiquitin-conjugating enzyme genes participating in ubiquitination of abnormal proteins under low-temperature stress. Moreover, when plants are under high salt and drought stress, endogenous ABA levels increase and a series of responses relying on ABA are activated (Christmann et al., 2006; Zhang et al., 2007; Santiago et al., 2009; Saavedra et al., 2010). In the ABA treatment, NnUBC3 showed upregulated expression, indicating that $\mathrm{UBC}$ has an important role in the ABA signal pathway. In A. thaliana, UBL has been shown to play an important role under high salt, drought, and ABA treatments (Zhang et al., 2007). Therefore, the role of UBC in N. nucifera, whether assisting the UBL degradation of proteins or mono-ubiquitination of the gene at a certain site to activate the ABA signal pathway, needs to be further studied.

In this study, we cloned four UBC genes in lotus. These genes were widely expressed in the lotus organism, indicating that they contribute to multiple biological processes. Among them, NnUBC3 was shown to take part in the control of flowering time, plant development, and response to drought and salt stress. Because the lotus is a basal dicotyledonous plant, these results will be helpful to further dissect the function of UBCs in lotus as well as the evolution of UBC genes in plants.

\section{Conflicts of interest}

The authors declare no conflict of interest.

\section{ACKNOWLEDGMENTS}

Research supported by the National Key Technologies R\&D Program

Genetics and Molecular Research 15 (3): gmr.15038341 
(\#2012BAD27B01). The authors thank the anonymous referees for their critical comments that contributed toward improving this manuscript.

\section{REFERENCES}

Bachmair A, Novatchkova M, Potuschak T and Eisenhaber F (2001). Ubiquitylation in plants: a post-genomic look at a post-translational modification. Trends Plant Sci. 6: 463-470. http://dx.doi.org/10.1016/S1360-1385(01)02080-5

Bae H and Kim WT (2014). Classification and interaction modes of 40 rice E2 ubiquitin-conjugating enzymes with 17 rice ARM-U-box E3 ubiquitin ligases. Biochem. Biophys. Res. Commun. 444: 575-580. http://dx.doi.org/10.1016/j. bbrc.2014.01.098

Bartel B and Citovsky V (2012). Focus on ubiquitin in plant biology. Plant Physiol. 160: 1. http://dx.doi.org/10.1104/ pp.112.203208

Borsch T and Barthlott W (1994). Classification and distribution of the genus Nelumbo Adans. (Nelumbonaceae). Beitr. Biol. Pflanzen 68: 421-450.

Cao Y, Dai Y, Cui S and Ma L (2008). Histone H2B monoubiquitination in the chromatin of FLOWERING LOCUS C regulates flowering time in Arabidopsis. Plant Cell 20: 2586-2602. http://dx.doi.org/10.1105/tpc.108.062760

Cheng YT and Li X (2012). Ubiquitination in NB-LRR-mediated immunity. Curr. Opin. Plant Biol. 15: 392-399. http:// dx.doi.org/10.1016/j.pbi.2012.03.014

Christmann A, Moes D, Himmelbach A, Yang Y, et al. (2006). Integration of abscisic acid signalling into plant responses. Plant Biol (Stuttg) 8: 314-325. http://dx.doi.org/10.1055/s-2006-924120

Clough SJ and Bent AF (1998). Floral dip: a simplified method for Agrobacterium-mediated transformation of Arabidopsis thaliana. Plant J. 16: 735-743. http://dx.doi.org/10.1046/j.1365-313x.1998.00343.x

Dikic I, Wakatsuki S and Walters KJ (2009). Ubiquitin-binding domains - from structures to functions. Nat. Rev. Mol. Cell Biol. 10: 659-671. http://dx.doi.org/10.1038/nrm2767

Dreher K and Callis J (2007). Ubiquitin, hormones and biotic stress in plants. Ann. Bot. (Lond.) 99: 787-822. http://dx.doi. org/10.1093/aob/mcl255

Esau K and Kosakai H (1975). Leaf arrangement in Nelumbo nucifera: a re-examination of a unique phyllotaxy. Phytomorphology 25: 100-112.

Gu X, Jiang D, Wang Y, Bachmair A, et al. (2009). Repression of the floral transition via histone H2B monoubiquitination. Plant J. 57: 522-533. http://dx.doi.org/10.1111/j.1365-313X.2008.03709.x

Ingvardsen C and Veierskov B (2001). Ubiquitin- and proteasome-dependent proteolysis in plants. Physiol. Plant. 112: 451-459. http://dx.doi.org/10.1034/j.1399-3054.2001.1120401.x

Kraft E, Stone SL, Ma L, Su N, et al. (2005). Genome analysis and functional characterization of the E2 and RINGtype E3 ligase ubiquitination enzymes of Arabidopsis. Plant Physiol. 139: 1597-1611. http://dx.doi.org/10.1104/ pp.105.067983

Liu Y, Koornneef M and Soppe WJ (2007). The absence of histone H2B monoubiquitination in the Arabidopsis hub1 (rdo4) mutant reveals a role for chromatin remodeling in seed dormancy. Plant Cell 19: 433-444. http://dx.doi. org/10.1105/tpc. 106.049221

Livak KJ and Schmittgen TD (2001). Analysis of relative gene expression data using real-time quantitative PCR and the 2(-Delta Delta C(T)) Method. Methods 25: 402-408. http://dx.doi.org/10.1006/meth.2001.1262

Maier A, Schrader A, Kokkelink L, Falke C, et al. (2013). Light and the E3 ubiquitin ligase COP1/SPA control the protein stability of the MYB transcription factors PAP1 and PAP2 involved in anthocyanin accumulation in Arabidopsis. Plant J. 74: 638-651. http://dx.doi.org/10.1111/tpj.12153

Ming R, VanBuren R, Liu Y, Yang M, et al. (2013). Genome of the long-living sacred lotus (Nelumbo nucifera Gaertn.). Genome Biol. 14: R41. http://dx.doi.org/10.1186/gb-2013-14-5-r41

Moon J, Parry G and Estelle M (2004). The ubiquitin-proteasome pathway and plant development. Plant Cell 16: 31813195. http://dx.doi.org/10.1105/tpc. 104.161220

Pickart CM (2001). Mechanisms underlying ubiquitination. Annu. Rev. Biochem. 70: 503-533. http://dx.doi.org/10.1146/ annurev.biochem.70.1.503

Priestley DA and Posthumus MA (1982). Extreme longevity of lotus seeds from Pulantien. Nature 299: 148-149. http:// dx.doi.org/10.1038/299148a0

Saavedra X, Modrego A, Rodríguez D, González-García MP, et al. (2010). The nuclear interactor PYL8/RCAR3 of Fagus sylvatica $\mathrm{FsPP} 2 \mathrm{C} 1$ is a positive regulator of abscisic acid signaling in seeds and stress. Plant Physiol. 152: 133-150. http://dx.doi.org/10.1104/pp.109.146381

Genetics and Molecular Research 15 (3): gmr.15038341 
Santiago J, Rodrigues A, Saez A, Rubio S, et al. (2009). Modulation of drought resistance by the abscisic acid receptor PYL5 through inhibition of clade A PP2Cs. Plant J. 60: 575-588. http://dx.doi.org/10.1111/j.1365-313X.2009.03981.x

Scheffner M, Nuber U and Huibregtse JM (1995). Protein ubiquitination involving an E1-E2-E3 enzyme ubiquitin thioester cascade. Nature 373: 81-83. http://dx.doi.org/10.1038/373081a0

Shi SQ, Shi Z, Jiang ZP, Qi LW, et al. (2010). Effects of exogenous GABA on gene expression of Caragana intermedia roots under $\mathrm{NaCl}$ stress: regulatory roles for $\mathrm{H}_{2} \mathrm{O}_{2}$ and ethylene production. Plant Cell Environ. 33: 149-162. http:// dx.doi.org/10.1111/j.1365-3040.2009.02065.x

Smalle J and Vierstra RD (2004). The ubiquitin 26S proteasome proteolytic pathway. Annu. Rev. Plant Biol. 55: 555-590. http://dx.doi.org/10.1146/annurev.arplant.55.031903.141801

Sridhar KR and Bhat R (2007). Lotus - A potential nutraceutical source. J. Agric. Technol 3: 143-155.

van Wijk SJ and Timmers HT (2010). The family of ubiquitin-conjugating enzymes (E2s): deciding between life and death of proteins. FASEB J. 24: 981-993. http://dx.doi.org/10.1096/fj.09-136259

Xu L, Ménard R, Berr A, Fuchs J, et al. (2009). The E2 ubiquitin-conjugating enzymes, AtUBC1 and AtUBC2, play redundant roles and are involved in activation of FLC expression and repression of flowering in Arabidopsis thaliana. Plant J. 57: 279-288. http://dx.doi.org/10.1111/j.1365-313X.2008.03684.x

Zhang Y, Yang C, Li Y, Zheng N, et al. (2007). SDIR1 is a RING finger E3 ligase that positively regulates stress-responsive abscisic acid signaling in Arabidopsis. Plant Cell 19: 1912-1929. http://dx.doi.org/10.1105/tpc.106.048488

Genetics and Molecular Research 15 (3): gmr.15038341 